\title{
Composition tuning of ultrafine cobalt-based spinel nanoparticles for
}

\section{efficient oxygen evolution}

Jingbo Han, ${ }^{a}$ Xiaohe Liu, ${ }^{a} *$ Hao Wan, ${ }^{a}$ Dan Wu, ${ }^{a}$ Gen Chen, ${ }^{a}$ Junhui Li,${ }^{a}$ Yijun Cao, ${ }^{b}$ Renzhi $M a^{c, *}$

${ }^{a}$ School of Materials Science and Engineering, Central South University, Changsha, Hunan 410083, P.R. China.

b Henan Province Industrial Technology Research Institute of Resources and Materials, Zhengzhou University, Zhengzhou, Henan 450001, P. R. China.

c International Center for Materials Nanoarchitectonics (WPI-MANA), National Institute for Materials Science (NIMS), Tsukuba, Ibaraki 305-0044, Japan.

\section{Corresponding author}

*Email: liuxh@csu.edu.cn (X. Liu)

*Email: MA.Renzhi@nims.go.jp (R. Ma)

\begin{tabular}{cc|cc}
\hline Content & Page No. & Content & Page No. \\
\hline Figure S1 & Page S2 & Figure S6 & Page S7 \\
Table S1 & Page S2 & Table S4 & Page S7 \\
Table S2 & Page S3 & Table S5 & Page S7 \\
Figure S2 & Page S3 & Figure S7 & Page S8 \\
Figure S3 & Page S4 & Figure S8 & Page S9 \\
Figure S4 & Page S4 & Figure S9 & Page S10 \\
Figure S5 & Page S5 & Figure S10 & Page S11 \\
Table S3 & Page S6 & & \\
\hline
\end{tabular}

Number of pages: 14

Number of figures: 10

Number of tables: 5 

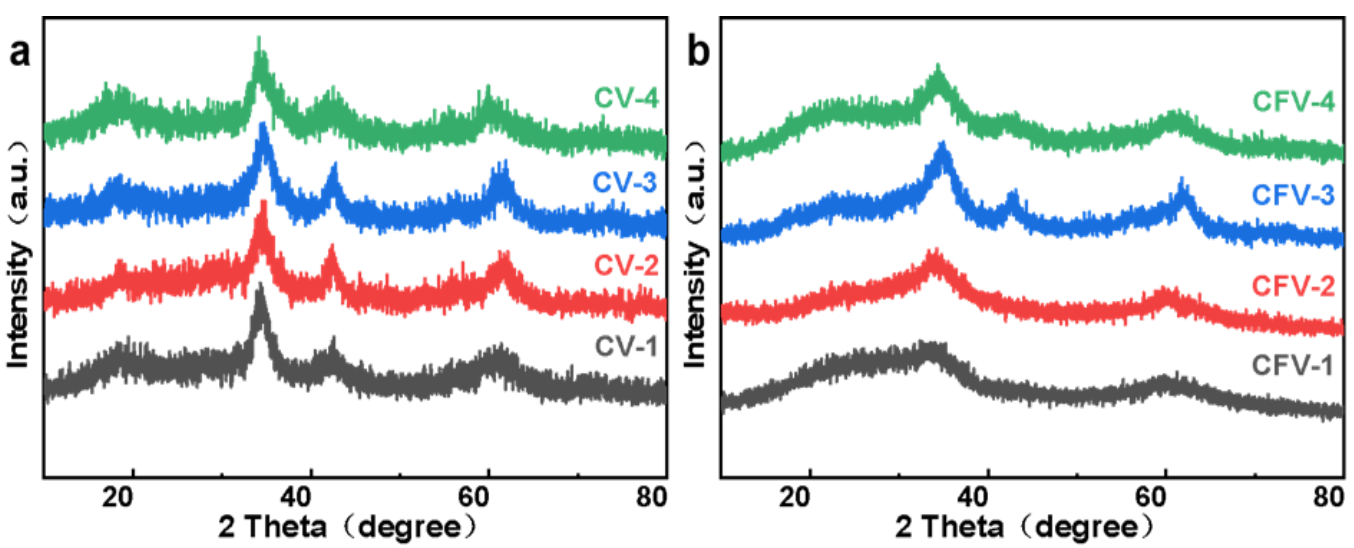

Figure S1. XRD patterns of (a) binary oxides (b) ternary oxides in different molar ratios.

Table S1. Composition analysis of the synthesized oxides by EDS.

\begin{tabular}{cc}
\hline Designed ratio $(\mathrm{Co}: \mathrm{Fe}: \mathrm{V})$ & Practical ratio $(\mathrm{Co}: \mathrm{Fe}: \mathrm{V})$ \\
\hline $1: 0: 0\left(\mathrm{Co}_{3} \mathrm{O}_{4}\right)$ & $1.31: 0: 1$ \\
$2: 0: 1(\mathrm{CV}-1)$ & $1.88: 0: 1$ \\
$3: 0: 1(\mathrm{CV}-2)$ & $2.77: 0: 1$ \\
$5: 0: 1(\mathrm{CV}-3)$ & $4.49: 0: 1$ \\
$10: 0: 1(\mathrm{CV}-4)$ & $1.49: 1.24: 1$ \\
$2: 1: 1(\mathrm{CFV}-1)$ & $1.83: 1.13: 1$ \\
$3: 1: 1(\mathrm{CFV}-2)$ & $2.81: 0.94: 1$ \\
$5: 1: 1(\mathrm{CFV}-3)$ & $4.68: 1.19: 1$ \\
$10: 1: 1(\mathrm{CFV}-4)$ &
\end{tabular}


Table S2. Interplanar spacings of 311 and 440 in synthesized $\mathrm{Co}_{3} \mathrm{O}_{4}, \mathrm{CV}-2$ and CFV-2.

\begin{tabular}{cccc}
\hline Catalyst & $\mathrm{d}_{311}(\mathrm{~nm})$ & $\mathrm{d}_{440}(\mathrm{~nm})$ & $\mathrm{a}(\mathrm{nm})$ \\
\hline $\mathrm{Co}_{3} \mathrm{O}_{4}$ & 0.244 & 0.143 & 0.809 \\
$\mathrm{CV}-2$ & 0.257 & 0.150 & 0.851 \\
$\mathrm{CFV}-2$ & 0.262 & 0.155 & 0.872 \\
\hline
\end{tabular}
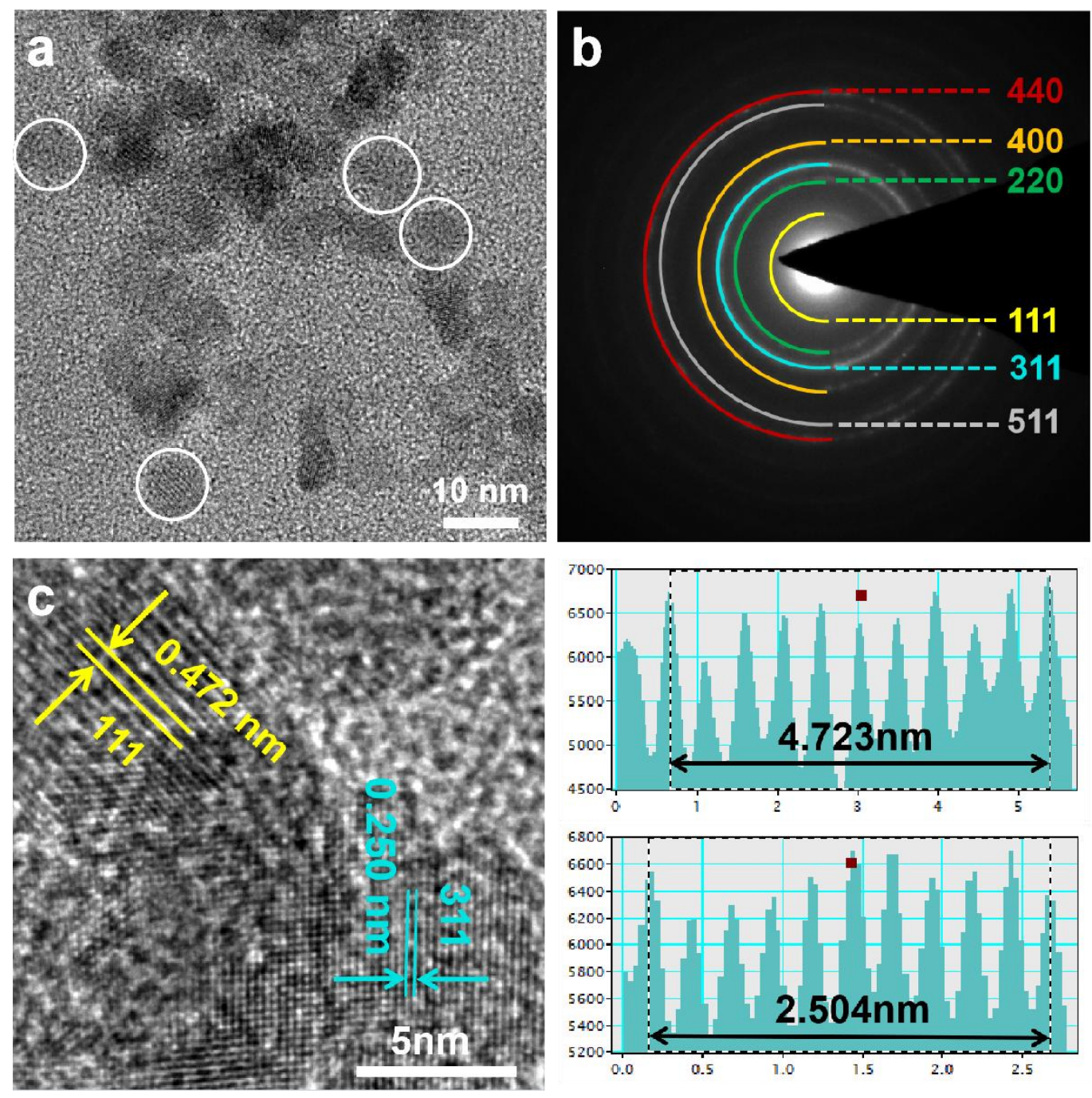

Figure S2. (a) TEM image (b) SAED pattern, (c) HRTEM image of as-synthesized $\mathrm{Co}_{3} \mathrm{O}_{4}$. The two different interplanar spacings coincide with those of 311 and 111 planes of $\mathrm{Co}_{3} \mathrm{O}_{4}$, respectively. 

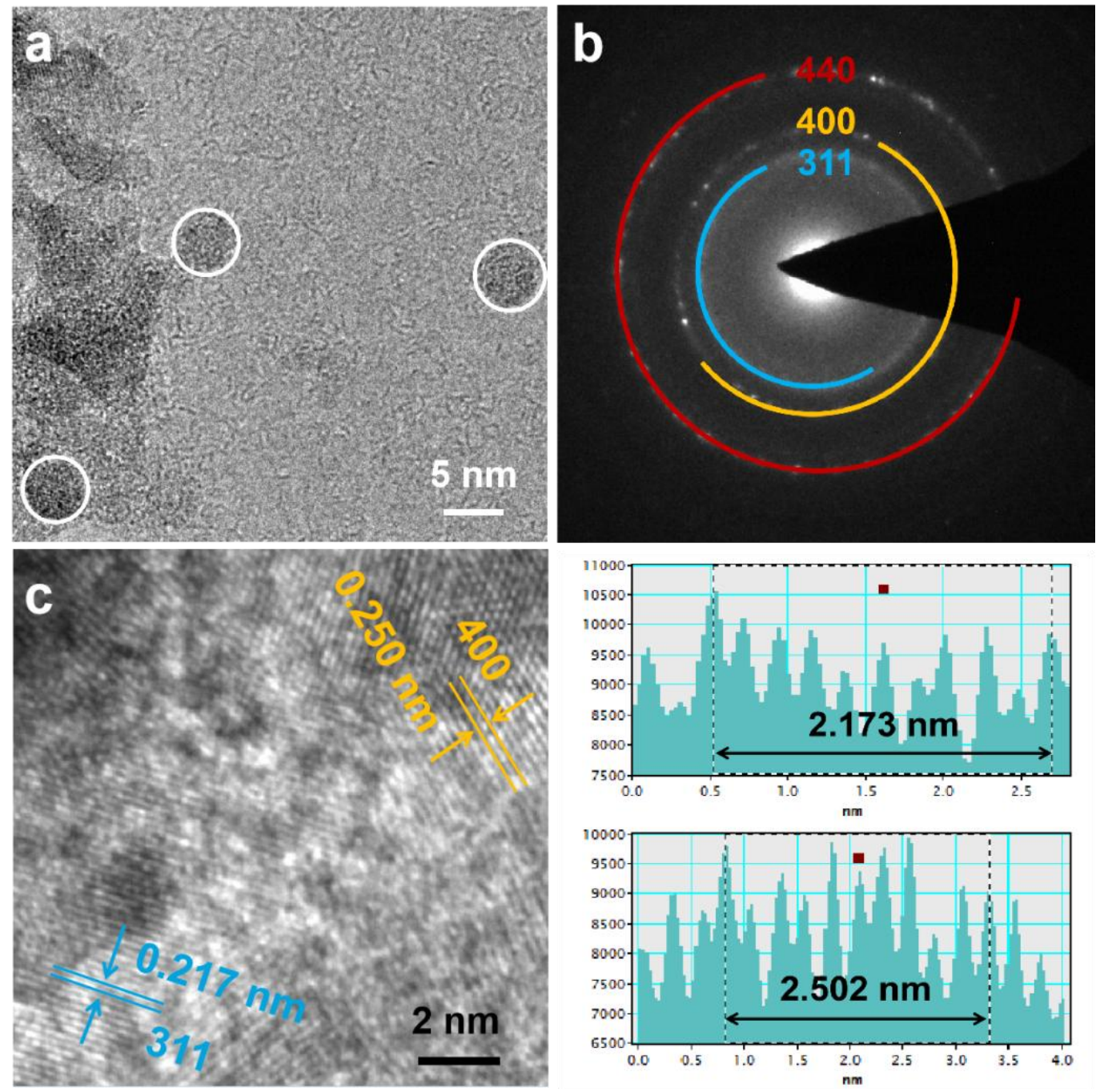

Figure S3. (a) TEM image (b) SAED pattern, (c) HRTEM image of as-synthesized CV-2. The two interplanar spacings in (c) respond to 311 and 400 planes of binary CoV spinel oxide, respectively.
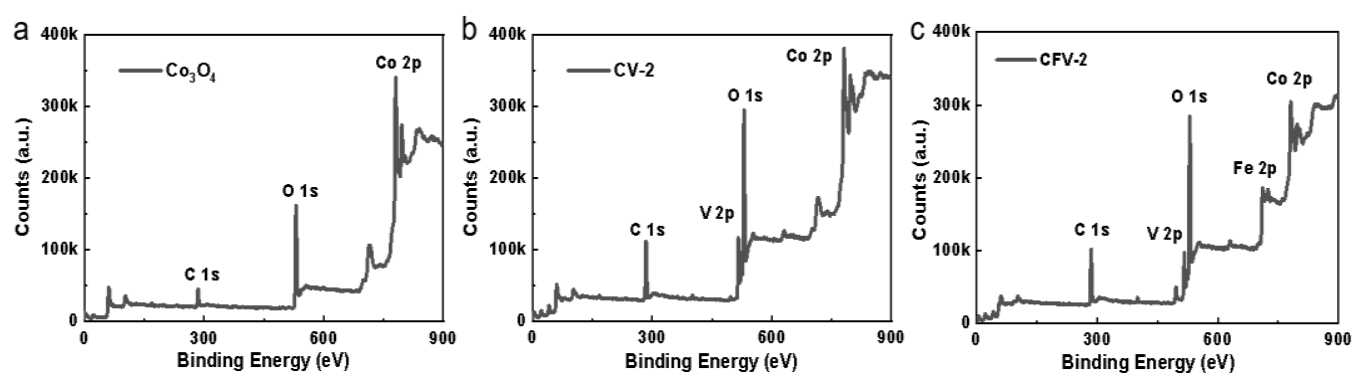

Figure S4. Wide-scan XPS spectra of (a) $\mathrm{Co}_{3} \mathrm{O}_{4}$, (b) CV-2 and (c)CFV-2. 

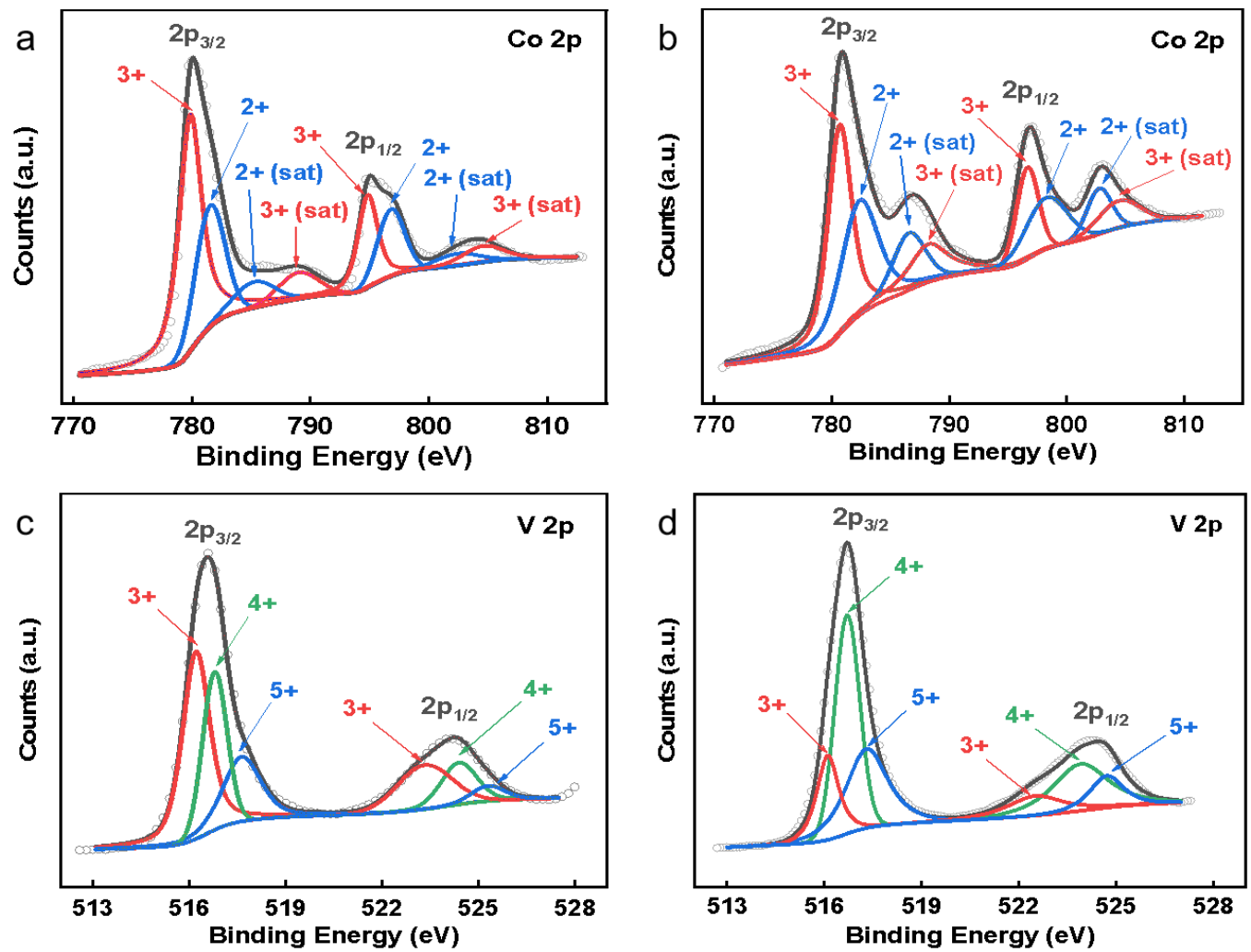

Figure S5. XPS spectra (a) Co $2 p$ of $\mathrm{Co}_{3} \mathrm{O}_{4}$, (b) Co $2 p$ of CV-2, (c) V 2p of CV-2 and (d) V $2 p$ of CFV-2. 
Table S3. Comparison of OER activity of as-synthesized oxides with reported electrocatalysts in the electrolyte of $1 \mathrm{M} \mathrm{KOH}$.

\begin{tabular}{|c|c|c|c|c|}
\hline Catalysts & Substrate & Overpotential & Tafel slope & Reference \\
\hline $\mathrm{CoFeVO}_{4}$ & Glassy carbon & 248 mV@10 mA cm ${ }^{-2}$ & $52.8 \mathrm{mV} \mathrm{dec}^{-1}$ & This work \\
\hline $\mathrm{Co}_{2} \mathrm{VO}_{4}$ & Glassy carbon & 295 mV@10 mA cm ${ }^{-2}$ & $65.9 \mathrm{mV} \mathrm{dec}{ }^{-1}$ & This work \\
\hline $\mathrm{Co}_{3} \mathrm{O}_{4}$ & Glassy carbon & 316mV@10 mA cm ${ }^{-2}$ & $78.6 \mathrm{mV} \mathrm{dec}{ }^{-1}$ & This work \\
\hline $\mathrm{Co}_{3} \mathrm{O}_{4} / \mathrm{N}-\mathrm{rmGO}$ & $\mathrm{Ni}$ foam & 310mV@10 mA cm ${ }^{-2}$ & $67 \mathrm{mV} \mathrm{dec}{ }^{-1}$ & 1 \\
\hline reduced $\mathrm{Co}_{3} \mathrm{O}_{4}$ & Glassy carbon & 420mV@13.1 mA cm ${ }^{-2}$ & $72 \mathrm{mV} \mathrm{dec}{ }^{-1}$ & 2 \\
\hline $\mathrm{P}-\mathrm{Co}_{3} \mathrm{O}_{4}$ & $\mathrm{Ni}$ foam & 280mV@10 mA cm ${ }^{-2}$ & $51.6 \mathrm{mV} \mathrm{dec}{ }^{-1}$ & 3 \\
\hline $\mathrm{Co}_{3} \mathrm{~V}_{2} \mathrm{O}_{8}$ & Glassy carbon & 359mV@10 mA cm ${ }^{-2}$ & $65 \mathrm{mV} \mathrm{dec}{ }^{-1}$ & 4 \\
\hline $\begin{array}{c}\text { reduced } \mathrm{CoFe}_{2} \mathrm{O}_{4} \\
\mathrm{NS}\end{array}$ & Glassy carbon & 320mV@10 mA cm ${ }^{-2}$ & $48 \mathrm{mV} \mathrm{dec}^{-1}$ & 5 \\
\hline $\mathrm{CoFe}-\mathrm{LDH}$ & $\mathrm{Ni}$ foam & 300mV@10 mA cm ${ }^{-2}$ & $83 \mathrm{mV} \mathrm{dec}^{-1}$ & 6 \\
\hline NiCoFe LTHs/CFC & $\begin{array}{l}\text { Carbon fiber } \\
\text { cloth }\end{array}$ & 239mV@10 mA cm ${ }^{-2}$ & $32 \mathrm{mV} \mathrm{dec}{ }^{-1}$ & 7 \\
\hline $\mathrm{Fe}$-doped $\mathrm{NiCo}_{2} \mathrm{O}_{4}$ & Ni foam & $350 \mathrm{mV} @ 10 \mathrm{~mA} \mathrm{~cm}{ }^{-2}$ & $27 \mathrm{mV} \mathrm{dec}{ }^{-1}$ & 8 \\
\hline CoFeZr oxides/NF & Ni foam & 264mV@20 mA cm ${ }^{-2}$ & $54.2 \mathrm{mV} \mathrm{dec}^{-1}$ & 9 \\
\hline NiV-LDH & Glassy carbon & $318 \mathrm{mV} @ 10 \mathrm{~mA} \mathrm{~cm}{ }^{-2}$ & $50 \mathrm{mV} \mathrm{dec}{ }^{-1}$ & 10 \\
\hline NiFe-LDH/rGO & Glassy carbon & 210mV@10 mA cm ${ }^{-2}$ & $40 \mathrm{mV} \mathrm{dec}^{-1}$ & 11 \\
\hline NiVFe-LDH & Ni foam & 231mV@10 mA cm ${ }^{-2}$ & $39.4 \mathrm{mV} \mathrm{dec}^{-1}$ & 12 \\
\hline NiVFe-LDH & Ni foam & $192 \mathrm{mV} @ 10$ mA cm ${ }^{-2}$ & $42 \mathrm{mV} \mathrm{dec}{ }^{-1}$ & 13 \\
\hline $\mathrm{VOOH}$ & $\mathrm{Ni}$ foam & 270mV@10 mA cm ${ }^{-2}$ & $68 \mathrm{mV} \mathrm{dec}^{-1}$ & 14 \\
\hline
\end{tabular}



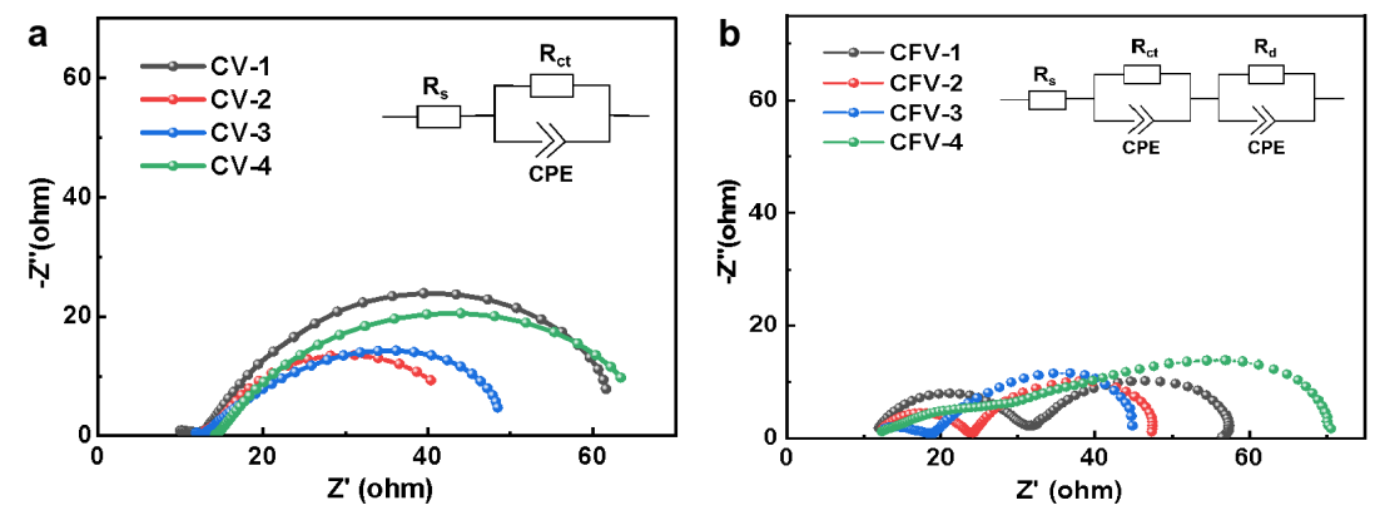

Figure S6. (a) EIS spectra of (a) binary oxides CV with different molar ratios, (b) ternary oxides CFV with different molar ratios. The inset of (a) and (b) are the corresponding equivalent circuits.

Table S4. The fitted values of resistances of $R_{s}$ and $R_{c t}$ of binary oxides.

\begin{tabular}{ccc}
\hline Catalyst & $\mathrm{R}_{\mathrm{s}}(\Omega)$ & $\mathrm{R}_{\mathrm{ct}}(\Omega)$ \\
\hline $\mathrm{CV}-1$ & 12.0 & 52.3 \\
$\mathrm{CV}-2$ & 11.5 & 32.5 \\
$\mathrm{CV}-3$ & 11.1 & 39.8 \\
$\mathrm{CV}-4$ & 11.8 & 55.2 \\
\hline
\end{tabular}

Table S5. The fitted values of resistances of $R_{s}, R_{c t}$ and $R_{d}$ of binary oxides.

\begin{tabular}{cccc}
\hline Catalyst & $\mathrm{R}_{\mathrm{s}}(\Omega)$ & $\mathrm{R}_{\mathrm{d}}(\Omega)$ & $\mathrm{R}_{\mathrm{ct}}(\Omega)$ \\
\hline CFV-1 & 11.2 & 19.9 & 28.5 \\
CFV-2 & 11.6 & 12.2 & 24.0 \\
CFV-3 & 10.9 & 13.1 & 26.9 \\
CFV-4 & 10.6 & 22.8 & 34.6 \\
\hline
\end{tabular}



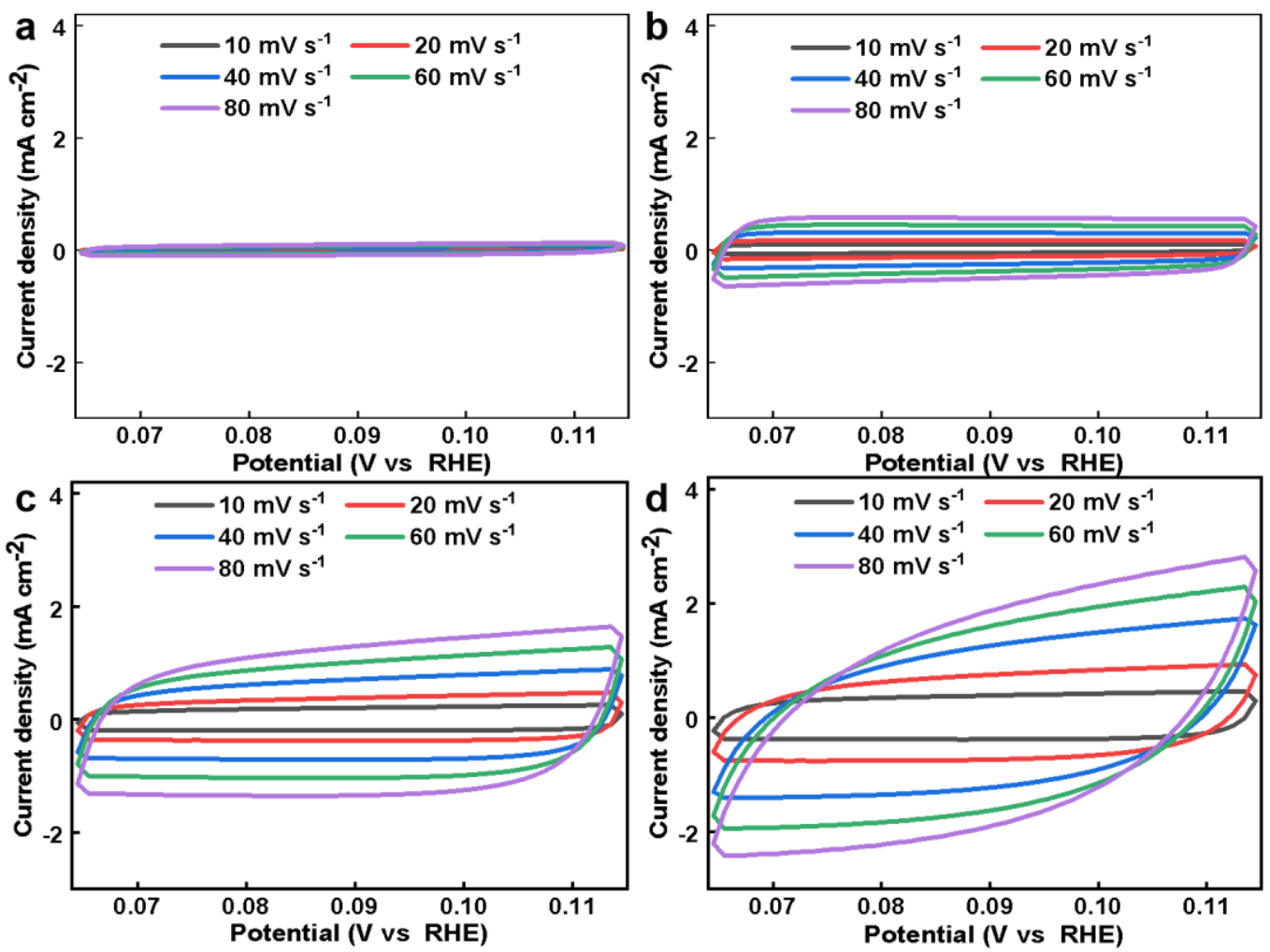

Figure S7. CV curves of (a) $\mathrm{Co}_{3} \mathrm{O}_{4}$ (b) $\mathrm{RuO}_{2}$ (c) CV-2 (d) CFV-2 at incremental scan rates. 

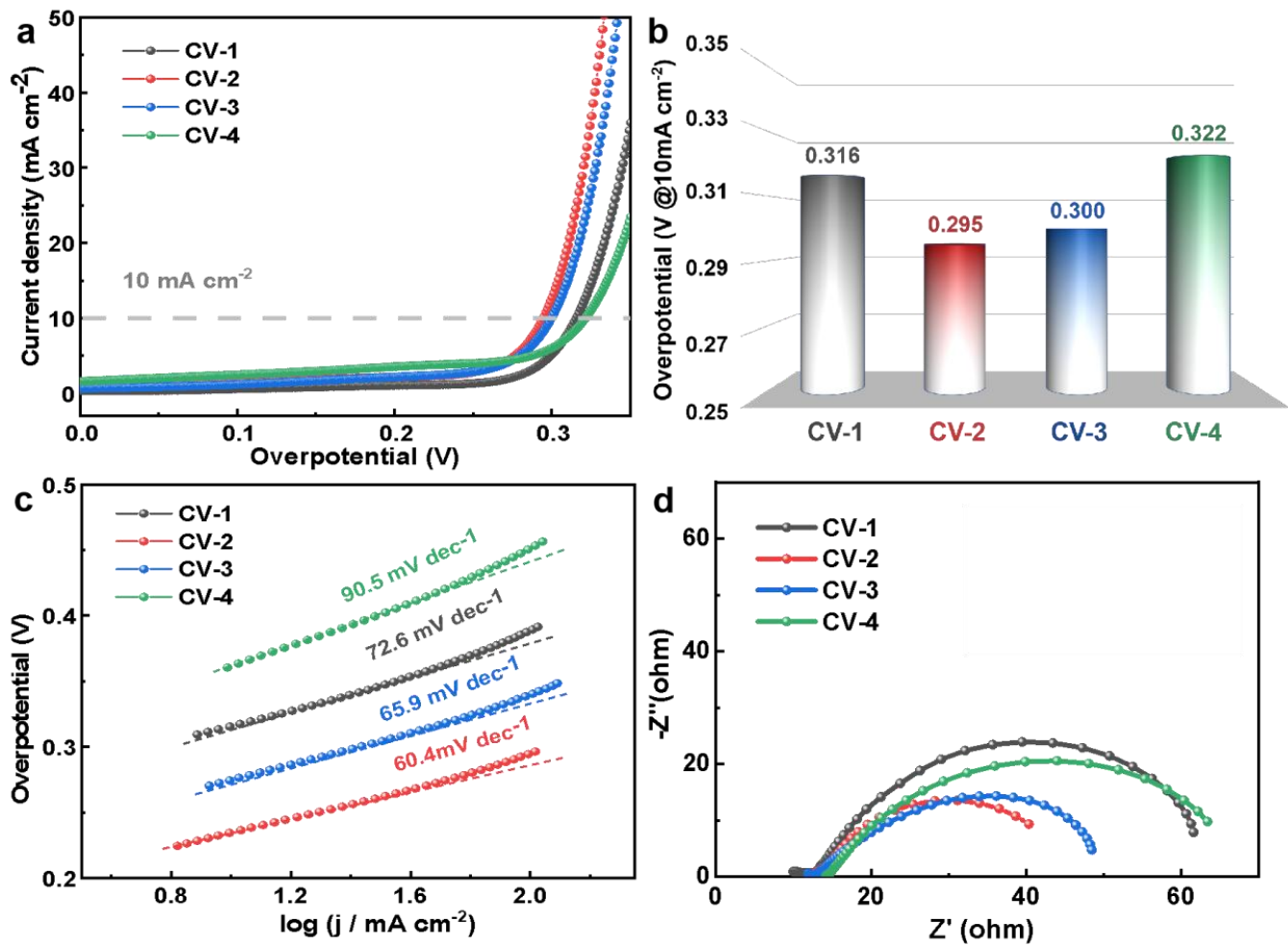

Figure S8. (a) LSV curves (b) Histogram of overpotential at $10 \mathrm{~mA} \mathrm{~cm}{ }^{-2}$ (c) Tafel slopes (d) EIS spectra of binary oxides CV with different molar ratios. 


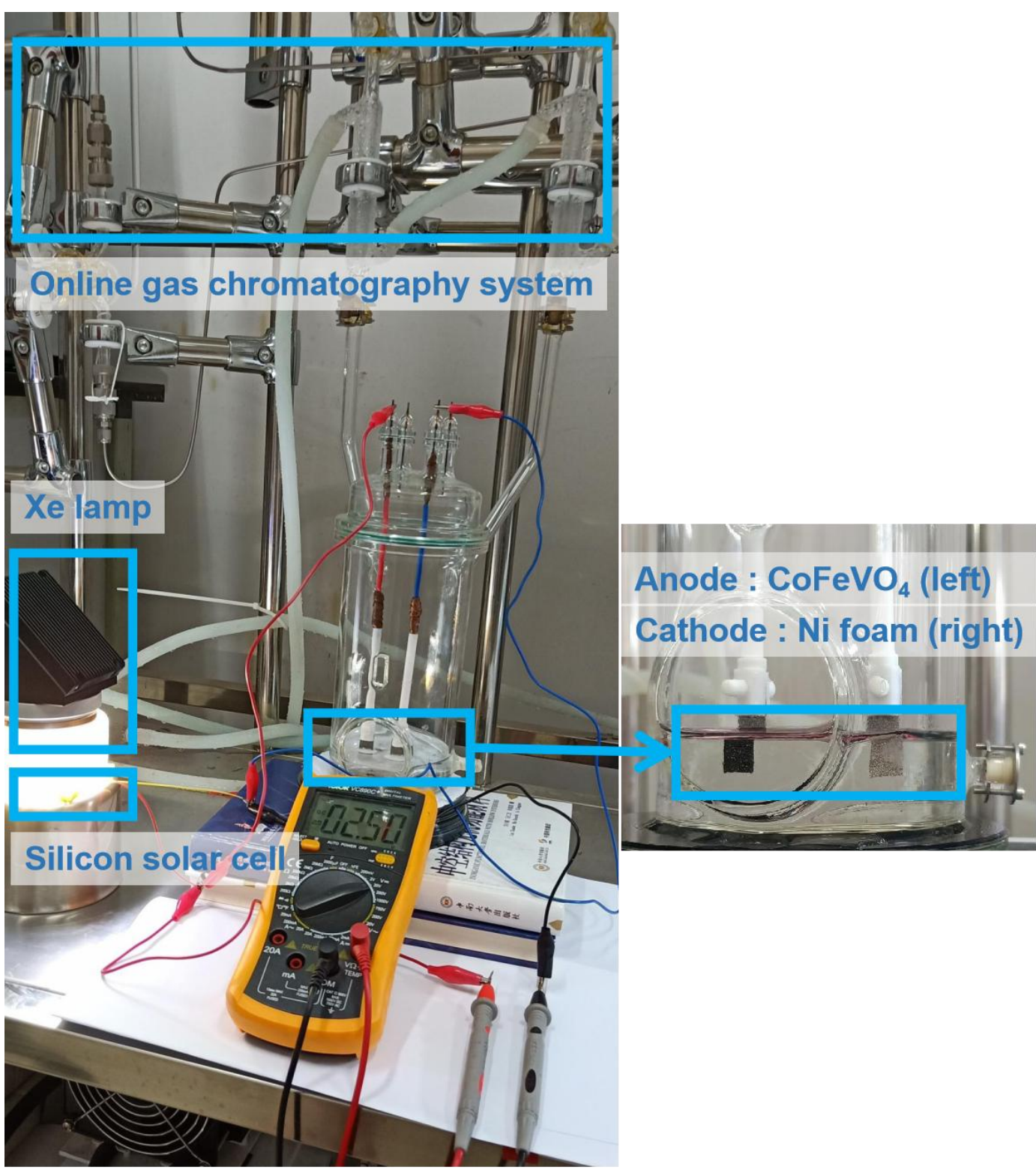

Figure S9. The photograph of the device.

Faraday efficiency was defined as the ratio between the actual and theoretical amount of the product. The total charge $(Q=I \times t)$ passed during the reaction was divided by $n \times F$ ( $n$ is the number of electron transfer, $F$ is the Faraday constant) to get the theoretical gas yield. $m$ is the actual moles of the product $(6122.5 \mu \mathrm{mol}$ during the first 1.5 hour). The calculation process for the Faraday efficiency is as following: Faraday Efficiency $=$ actual gas yield $/$ theoretical gas yield $=\mathrm{m} /[(\mathrm{I} \times \mathrm{t}) /(\mathrm{n} \times \mathrm{F})]=$ $(\mathrm{m} \times \mathrm{n} \times \mathrm{F}) /(\mathrm{I} \times \mathrm{t})=\left(6122.5 \times 10^{-6} \mathrm{~mol} \times 2 \times 96485 \mathrm{C} \mathrm{mol}^{-1}\right) /\left(224 \times 10^{-3} \mathrm{~A} \times 5400\right.$ s) $=97.7 \%$. 


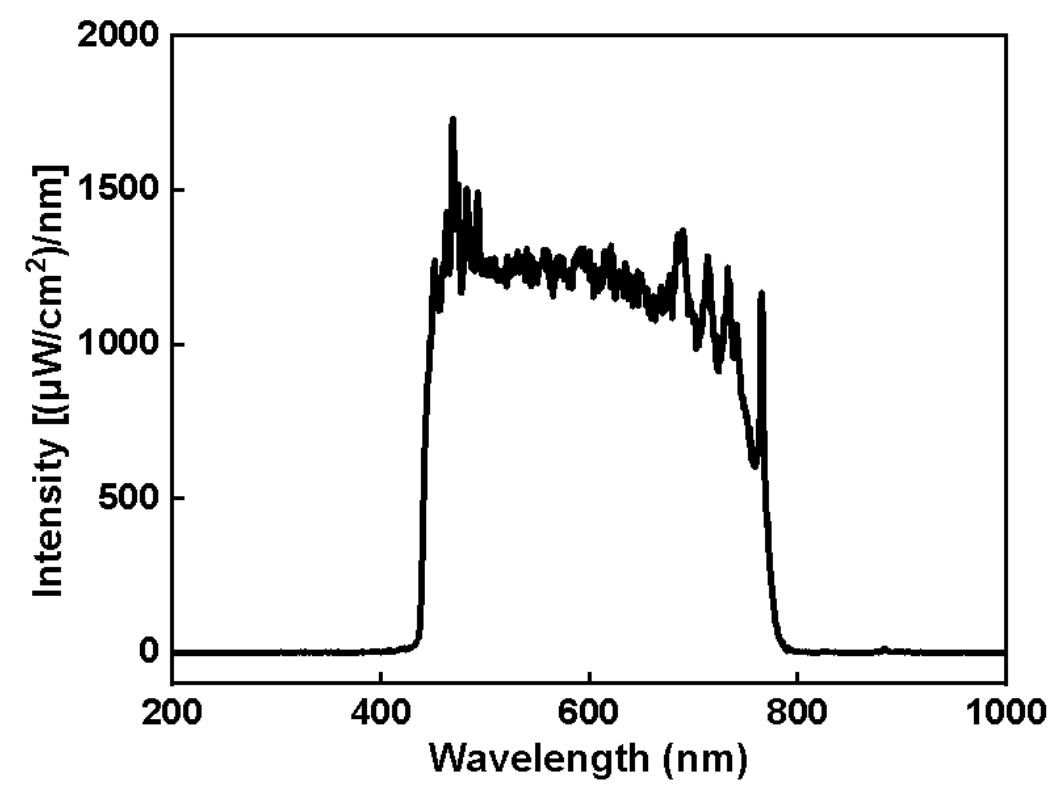

Figure S10. Irradiative spectrum of the Xe lamp (the major intensity is concentrated on the range of $400 \mathrm{~nm}<\lambda<800 \mathrm{~nm}$ ).

The average integral light intensity measured by the light meter is $0.3887 \mathrm{~W} \mathrm{~cm}^{-2}$. The irradiated area for the silicon solar cell is $14.4 \mathrm{~cm}^{2}$. The standard molar enthalpy of combustion for $\mathrm{H}_{2}$ is $-285.84 \mathrm{~kJ} \mathrm{~mol}^{-1}$. The yield of $\mathrm{H}_{2}$ during the first 1.5 hour is $6122.5 \mu \mathrm{mol}$. The calculation steps are as following:

Input: Solar energy $(\mathrm{J})=$ light intensity $\left(\mathrm{W} \mathrm{cm}^{-2}\right) \times$ illumination area $\left(\mathrm{cm}^{2}\right) \times$ time $(\mathrm{s})$ $=0.3887 \mathrm{~W} \mathrm{~cm}^{-2} \times 14.4 \mathrm{~cm}^{2} \times 5400 \mathrm{~s}=30.2253 \mathrm{~kJ}$ Output: $\mathrm{H}_{2}$ energy $(\mathrm{kJ})$ = standard molar enthalpy of combustion $\left(\mathrm{kJ} \mathrm{mol}^{-1}\right) \times \mathrm{H}_{2}$ moles $(\mathrm{mol})=285.84 \mathrm{~kJ} \mathrm{~mol}^{-1} \times 6122.5 \times 10^{-6} \mathrm{~mol}=1.7501 \mathrm{~kJ}$ Solar-to-Hydrogen energy conversion efficiency $=\mathrm{H}_{2}$ energy $(\mathrm{kJ}) /$ Solar energy $(\mathrm{kJ})$ $=1.7501 / 30.2253=5.8 \%$ 


\section{References}

(1) Liang, Y., Li, Y., Wang, H., Zhou, J., Wang, J., Regier, T., Dai, H. $\mathrm{Co}_{3} \mathrm{O}_{4}$ nanocrystals on graphene as a synergistic catalyst for oxygen reduction reaction. Nat. Mater. 2011, 10(10), 780-786, DOI 10.1038/nmat3087.

(2) Wang, Y., Zhou, T., Jiang, K., Da, P., Peng, Z., Tang, J., Zheng, G. Reduced mesoporous $\mathrm{Co}_{3} \mathrm{O}_{4}$ nanowires as efficient water oxidation electrocatalysts and supercapacitor electrodes. Adv. Energy Mater. 2014,4(16), 1400696, DOI 10.1002/aenm.201400696.

(3) Xiao, Z., Wang, Y., Huang, Y. C., Wei, Z., Dong, C. L., Ma, J., Wang, S. Filling the oxygen vacancies in $\mathrm{Co}_{3} \mathrm{O}_{4}$ with phosphorus: an ultra-efficient electrocatalyst for overall water splitting. Energy Environ. Sci. 2017, 10(12), 2563-2569, DOI 10.1039/c7ee01917c.

(4) Xing, M., Kong, L. B., Liu, M. C., Liu, L. Y., Kang, L., Luo, Y. C. Cobalt vanadate as highly active, stable, noble metal-free oxygen evolution electrocatalyst. J. Mater. Chem. A 2014,2(43), 18435-18443, DOI 10.1039/C4TA03776F.

(5) Yan, K., Shang, X., Liu, Z., Dong, B., Lu, S., Chi, J., Gao, W., Chai, Y., Liu, C. A facile method for reduced $\mathrm{CoFe}_{2} \mathrm{O}_{4}$ nanosheets with rich oxygen vacancies for efficient oxygen evolution reaction. Int. J. Hydrogen Energy 2017, 42(38), 24150-24158, DOI 10.1016/j.ijhydene.2017.07.165. 
(6) Feng, L., Li, A., Li, Y., Liu, J., Wang, L., Huang, L., Ge, X. A highly active CoFe layered double hydroxide for water splitting. ChemPlusChem 2017, 82(3), 483-488, DOI 10.1002/cplu.201700005.

(7) Wang, A. L., Xu, H., Li, G. R. NiCoFe layered triple hydroxides with porous structures as high-performance electrocatalysts for overall water splitting. ACS Energy Lett. 2016, 1(2), 445-453, DOI 10.1021/acsenergylett.6b00219.

(8) Yan, K. L., Shang, X., Li, Z., Dong, B., Li, X., Gao, W. K., Liu, C. G. Ternary mixed metal Fe-doped $\mathrm{NiCo}_{2} \mathrm{O}_{4}$ nanowires as efficient electrocatalysts for oxygen evolution reaction. Appl. Surf. Sci. 2017, 416, 371-378, DOI 10.1016/j.apsusc.2017.04.204.

(9) Huang, L., Chen, D., Luo, G., Lu, Y. R., Chen, C., Zou, Y., Wang, S. Zirconium-Regulation-Induced Bifunctionality in 3D Cobalt-Iron Oxide Nanosheets for Overall Water Splitting. Adv. Mater. 2019, 28(31), 1901439, DOI 10.1002/adma.201901439.

(10) Fan, K., Chen, H., Ji, Y., Huang, H., Claesson, P. M., Daniel, Q., Sun, L. Nickel-vanadium monolayer double hydroxide for efficient electrochemical water oxidation. Nat. Commun. 2016, 7, 11981, DOI 10.1038/ncomms11981.

(11) Ma, W., Ma, R., Wang, C., Liang, J., Liu, X., Zhou, K., Sasaki, T. A superlattice of alternately stacked $\mathrm{Ni}-\mathrm{Fe}$ hydroxide nanosheets and graphene for efficient splitting of water. ACS Nano 2015, 9(2), 1977-1984, DOI 10.1021/nn5069836.

(12) Dinh, K. N., Zheng, P., Dai, Z., Zhang, Y., Dangol, R., Zheng, Y., Yan, Q. Ultrathin porous $\mathrm{NiFeV}$ ternary layer hydroxide nanosheets as a highly efficient 
bifunctional electrocatalyst for overall water splitting. Small 2018, 14(8), 1703257, DOI 10.1002/smll.201703257.

(13) Li, P., Duan, X., Kuang, Y., Li, Y., Zhang, G., Liu, W., Sun, X. Tuning electronic structure of NiFe layered double hydroxides with vanadium doping toward high efficient electrocatalytic water oxidation. Adv. Energy Mater. 2018, 8(15), 1703341, DOI 10.1002/aenm.201703341.

(14) Shi, H., Liang, H., Ming, F., Wang, Z. Efficient Overall Water-Splitting Electrocatalysis Using Lepidocrocite VOOH Hollow Nanospheres. Angew. Chem. Int. Ed. 2017, 56(2), 573-577, DOI 10.1002/anie.201610211. 